Fixed Point Theory, 22(2021), No. 1, 391-406

DOI: $10.24193 /$ fpt-ro.2021.1.27

http://www.math.ubbcluj.ro/ nodeacj/sfptcj.html

\title{
COINCIDENCE POINTS FOR SET-VALUED MAPPINGS WITH DIRECTIONAL REGULARITY
}

\author{
BINBIN ZHANG* AND WEI OUYANG** \\ *School of Science, Kunming University of Science and Technology, \\ Kunming 650500, People's Republic of China \\ E-mail: bbzhang@kust.edu.cn \\ ** School of Mathematics, Yunnan Normal University, \\ Kunming 650500, People's Republic of China \\ E-mail: weiouyangxe@hotmail.com
}

\begin{abstract}
This paper is devoted to investigate the interrelations between directional metric regularity and coincidence points for set-valued mappings. Under the assumption of directional metric regularity and directional Aubin continuity, new coincidence point theorems were established through iteration procedures for both local and global cases. As an application, the (global) directional Aubin continuity for the solution mapping of partial-parametrized variational system was established.

Key Words and Phrases: Coincidence point, directional metric regularity, directional Aubin continuity, variational system.
\end{abstract}

2020 Mathematics Subject Classification: 49J53, 47H10, 54H25, 90C31.

Acknowledgement. The authors would like to express their gratitude to the referees for the helpful comments and suggestions on the original submission.

Binbin Zhang's research was supported by the National Natural Science Foundation of the People's Republic of China (grant 11771384), the Yunnan Provincial Department of Education Research Fund (grant 2019J0040) and the Fund for Fostering Talents in Kunming University of Science and Technology (No. KKSY 201807022).

Wei Ouyang's research was supported by the National Natural Science Foundation of the People's Republic of China (grant 11801500)

\section{REFERENCES}

[1] S. Adly, A.L. Dontchev, M. Théra, On one-sided Lipschitz stability of set-valued contractions, Numerical Functional Analysis and Optimization, 35(2014), 837-850.

[2] F.J. Aragon Artacho, B.S. Mordukhovich, Metric regularity and Lipscithian stability of parametric variational systems, Nonlinear Anal., 72(2010), 1149-1170.

[3] A.V. Arutyunov, Covering mappings in metric spaces and fixed points, Doklady Mathematics, 76(2007), 665-668.

[4] A.V. Arutyunov, Stability of coincidence points and properties of covering mappings, Mathematical Notes, 76(2009), 153-158.

[5] A.V. Arutyunov, E.R. Avakov, A.F. Izmailov, Directional regularity and metric regularity, SIAM J. Optim., 18(2007), 810-833. 
[6] A.V. Arutyunov, A.F. Izmailov, Directional stability theorem and directional metric regularity, Math. Oper. Res., 31(2006), 526-543.

[7] A.V. Arutyunov, E.S. Zhukovskiy, S.E. Zhukovskiy, Coincidence points principle for mappings in partially ordered spaces, Topology and its Applications, 179(2015), 13-33.

[8] A.V. Arutyunov, E.S. Zhukovskiy, S.E. Zhukovskiy, Coincidence points principle for set-valued mappings in partially ordered spaces, Topology and its Applications, 201(2016), 330-343.

[9] A.V. Arutyunov, E.S. Zhukovskiy, S.E. Zhukovskiy, Covering mappings and well-posedness of nonlinear Volterra equations, Nonlinear Anal., 75(2012), 1026-1044.

[10] E.R. Avakov, A.V. Arutyunov, S.E. Zhukovskiy, Stability theorems for estimating the distance to a set of coincidence points, SIAM J. Optim., 25(2015), 807-828.

[11] E.R. Avakov, A.V. Arutyunov, E.S. Zhukovskii, Covering mappings and their applications to differential equations unsolved for the derivative, Differential Equations, 45(2009), 627-649.

[12] G. Beer, A.L. Dontchev, The weak Ekeland variational principle and fixed points, Nonlinear Anal., 102(2014), 91-96.

[13] M. Bianchi, G. Kassay, R. Pini, Stability results of variational systems under openness with respect to fixed sets, J. Optim. Theory Appl., 164(2015), 92-108.

[14] A.L. Dontchev, H. Frankowska, Lyusternik-Graves theorem and fixed points, Proc. Amer. Math. Soc., 139(2011), 521-534.

[15] A.L. Dontchev, R.T. Rockafellar, Implicit Functions and Solution Mappings, Springer, Berlin, 2009.

[16] M. Durea, M. Panţiruc, R. Strugariu, Minimal time function with respect to a set of directions: basic properties and applications, Optim. Methods Softw., 31(2016), 535-561.

[17] M. Durea, M. Panţiruc, R. Strugariu, A new type of directional regularity for mappings and applications to optimization, SIAM J. Optim., 27(2017), 1204-1229.

[18] H. Gfrerer, On directional metric subregularity and second-order optimality conditions for a class of nonsmooth mathematical programs, SIAM J. Optim., 23(2013), 632-665.

[19] A.D. Ioffe, V.M. Tihomirov, Theory of Extremal Problems, Nauka, Moscow, 1974 (in Russian); English transl.: North-Holland, Amsterdam, 1979.

[20] A.D. Ioffe, Towards variational analysis in metric spaces: metric regularity and fixed points, Math. Program., Serie B, 123(2010), 241-252.

[21] A.D. Ioffe, Regularity on a fixed set, SIAM J. Optim., 21(2011), 1345-1370.

[22] A.D. Ioffe, Metric regularity, fixed points and some associated problems of variational analysis, J. Fixed Point Theory Appl., 15(2014), 67-99.

[23] B.S. Mordukhovich, Variational Analysis and Generalized Differentiation, I. Basic Theory. II. Applications, Springer-Verlag, Berlin, 2006.

[24] S.B. Nadler, Jr., Multi-valued contraction mapping, Pacific J. Math., 30(1969), 475-488.

[25] N.M. Nam, C. Zalinescu, Variational analysis of directional minimal time functions and applications to location problems, Set-Valued Var. Anal., 21(2013), 405-430.

[26] H.V. Ngai, M. Théra, Directional metric regularity of multifunctions, Math. Oper. Res., 40(2015), 969-991.

Received: November 7, 2018; Accepted: December 17, 2019. 
